\title{
SOME APPLICATIONS OF SELECTION THEOREMS TO PARAMETRIZATION PROBLEMS
}

\author{
A. MAITRA, B. V. RAO AND V. V. SRIVATSA
}

(Communicated by R. Daniel Mauldin)

\begin{abstract}
Some results relating to parametrizations of Borel sets are deduced from selection theorems.
\end{abstract}

1. Introduction. Several authors, most notably Cenzer and Mauldin [1] and Mauldin [6], have shown that measurable parametrizations of Borel sets in the product of two Polish spaces can be constructed in two steps. Step 1 consists in the construction of homeomorphisms from the Cantor set into the sections of the Borel set, while step 2 involves carrying out a Schröder-Bernstein type argument. In this paper we will be concerned with the first of these steps. We take as our point of departure a recent article of Pol [7], wherein he used the selection theorem of von Neumann and Yankov to get hold of the homeomorphisms. Pol obtained analytically measurable parametrizations. We show that Pol's method admits of sufficient precision to yield Borel parametrizations. Incidentally, the present article provides new applications of Borel selection theorems.

The essence of Pol's method for obtaining an analytically measurable parametrization was to use the von Neumann-Yankov selection theorem to prove the following.

THEOREM 0. Let $X$ be a separable metric space, $F$ a multifunction from $X$ to $\omega^{\omega}$ such that $\operatorname{Graph}(F)$ is analytic in $X \times \omega^{\omega}$ and $F(x)$ is a nonempty, perfect subset of $\omega^{\omega}$ for each $x \in X$. Then there is a function $g: X \times 2^{\omega} \rightarrow \omega^{\omega}$ such that

(a) $g(x, \cdot)$ is a homeomorphism of $2^{\omega}$ into $F(x)$ for each $x \in X$, and

(b) $g(\cdot, y)$ is analytically measurable on $X$ for each $y \in 2^{\omega}$.

In the same spirit and as illustrative of the power of Pol's method, we shall prove the following results in the present paper.

THEOREM 1. Let $X$ be a separable metric space, $F$ a Borel measurable multifunction from $X$ to $\omega^{\omega}$ such that $F(x)$ is a nonempty, perfect subset of $\omega^{\omega}$ for each $x \in X$. Then there is a function $g: X \times 2^{\omega} \rightarrow \omega^{\omega}$ such that

(a) $g(x, \cdot)$ is a homeomorphism of $2^{\omega}$ into $F(x)$ for each $x \in X$, and

(b) $g(\cdot, y)$ is Borel measurable on $X$ for each $y \in 2^{\omega}$.

THEOREM 2. Let $X$ be a separable metric space, $F$ a Borel measurable multifunction from $X$ to $2^{\omega}$ such that $F(x)$ is a nonempty, perfect subset of $2^{\omega}$ for each

Received by the editors June 11, 1987.

1980 Mathematics Subject Classification (1985 Revision). Primary 28A20, 54C65; Secondary $04 \mathrm{~A} 15$.

Key words and phrases. Borel measurable selections and parametrizations. 
$x \in X$. Then there is a function $g: X \times 2^{\omega} \rightarrow 2^{\omega}$ such that

(a) $g(x, \cdot)$ is a homeomorphism of $2^{\omega}$ onto $F(x)$ for each $x \in X$, and

(b) $g(\cdot, y)$ is Borel measurable on $X$ for each $y \in 2^{\omega}$.

In [5] it is shown that Borel parametrization theorems for Borel sets with large (vertical) sections can be reduced to Theorem 1 above. We shall use a selection theorem for $G_{\delta}$-valued multifunctions to prove Theorem 1 . Theorem 2 is a generalization of Proposition 1 of Purves [8], which was one of the early results in this area. We deduce Theorem 2 from the well-known Kuratowski-Ryll-Nardzewski selection theorem for closed-valued multifunctions.

The next section is devoted to some preliminaries and the proofs will be given in $\S 3$. We conclude with some remarks in $\S 4$.

2. Preliminaries. We fix some notation first. We denote the set of natural numbers by $\omega$. The set of infinite sequences of natural numbers (0's and 1's) is denoted by $\omega^{\omega}\left(2^{\omega}\right)$. Endowed with the product of discrete topologies on $\omega(\{0,1\})$, $\omega^{\omega}\left(2^{\omega}\right)$ becomes a homeomorph of the irrationals (Cantor set). If $u \in 2^{\omega}$ and $i \in \omega$, $u_{i}$ will denote the $i$ th coordinate of $u$. Define a metric $d$ on $2^{\omega}$ as follows:

$$
\begin{aligned}
d(u, v) & =1 /(k+1) & & \text { if } u \neq v \text { and } k \text { is the least } i \text { such that } u_{i} \neq v_{i}, \\
& =0 & & \text { if } u=v .
\end{aligned}
$$

Plainly, $d$ induces on $2^{\omega}$ the product of discrete topologies on $\{0,1\}$. For each finite sequence $s$ of 0 's and 1's, $P(s)$ will denote the set of elements of $2^{\omega}$ which start with $s$. Denote by $S_{k}$ the set of finite sequences of 0 's and 1's of length $k$.

The set of continuous functions on $2^{\omega}$ into $2^{\omega}$ is denoted by $C\left(2^{\omega}, 2^{\omega}\right)$. Equipped with the compact-open topology (or, equivalently, the topology of uniform convergence), $C\left(2^{\omega}, 2^{\omega}\right)$ becomes a Polish space. Let $\tilde{d}$ be the uniform metric induced on $C\left(2^{\omega}, 2^{\omega}\right)$ by $d$, that is, for $h, h^{\prime} \in C\left(2^{\omega}, 2^{\omega}\right)$,

$$
\tilde{d}\left(h, h^{\prime}\right)=\sup \left\{d\left(h(u), h^{\prime}(u)\right): u \in 2^{\omega}\right\} .
$$

Let $H\left(2^{\omega}\right)$ denote the set of homeomorphisms of $2^{\omega}$ into $2^{\omega}$. Now $H\left(2^{\omega}\right)$ is a $G_{\delta}$ in $C\left(2^{\omega}, 2^{\omega}\right)$. (Reason: For $h \in C\left(2^{\omega}, 2^{\omega}\right)$, we have

$$
\begin{aligned}
& h \in H\left(2^{\omega}\right) \leftrightarrow(\forall s)(\forall t)[P(s) \cap P(t) \\
&=\varnothing \rightarrow(\exists V)\left(\exists V^{\prime}\right)\left(V, V^{\prime} \text { are open in } 2^{\omega} \& V \cap V^{\prime}\right.=\varnothing \\
&\left.\left.\quad \& h(P(s)) \subseteq V \& h(P(t)) \subseteq V^{\prime}\right)\right] .
\end{aligned}
$$

Consequently, $H\left(2^{\omega}\right)$ with the topology inherited from $C\left(2^{\omega}, 2^{\omega}\right)$ is itself a Polish space. For fixed $y \in 2^{\omega}, e_{y}: H\left(2^{\omega}\right) \rightarrow 2^{\omega}$ denotes the evaluation map, that is, $e_{y}(h)=h(y)$. It is known that $e_{y}$ is continuous.

\section{Proofs.}

PROOF OF THEOREM 1. Imbed $\omega^{\omega}$ as a $G_{\delta}$ in $2^{\omega}$. So we may write $\omega^{\omega}=$ $\bigcap_{n \geq 0} G_{n}$, where each $G_{n}$ is open in $2^{\omega}$. Fix a countable dense set $D$ in $2^{\omega}$. We define a multifunction $\Phi$ from $X$ to $H\left(2^{\omega}\right)$ as follows:

$$
\Phi(x)=\left\{h \in H\left(2^{\omega}\right): h\left(2^{\omega}\right) \subseteq F(x)\right\} .
$$


Since $F(x)$ is a nonempty perfect subset of $\omega^{\omega}, \Phi(x)$ is nonempty. The graph of $\Phi$ is easily described thus:

$$
\begin{aligned}
\operatorname{Graph}(\Phi)= & {\left[X \times \bigcap_{n \geq 0}\left\{h \in H\left(2^{\omega}\right): h\left(2^{\omega}\right) \subseteq G_{n}\right\}\right] } \\
& \cap\left[\bigcap_{y \in D}\left\{(x, h) \in X \times H\left(2^{\omega}\right):\left(x, e_{y}(h)\right) \in \operatorname{Graph}(F)\right\}\right] .
\end{aligned}
$$

Since $F$ is Borel measurable and closed-valued in $\omega^{\omega}, \operatorname{Graph}(F)$ is a Borel set in $X \times \omega^{\omega}$ and hence Borel in $X \times 2^{\omega}$. It now follows that $\operatorname{Graph}(\Phi)$ is Borel in $X \times H\left(2^{\omega}\right)$. Moreover,

$$
\Phi(x)=\bigcap_{n \geq 0}\left\{h \in H\left(2^{\omega}\right): h\left(2^{\omega}\right) \subseteq G_{n}\right\} \cap \bigcap_{y \in D} e_{y}^{-1}(F(x)) .
$$

Recalling that $F(x)$ is closed in $\omega^{\omega}$ and so a $G_{\delta}$ in $2^{\omega}$, we see that $\Phi(x)$ is a $G_{\delta}$ in $H\left(2^{\omega}\right)$.

We now check that $\Phi$ is a Borel measurable multifunction. Let, then, $0<\eta<1$ and assume that $\eta \neq 1 / k, k \geq 1$. Fix $h^{*} \in H\left(2^{\omega}\right)$ and let $B$ be the open sphere in $H\left(2^{\omega}\right)$ with center $h^{*}$ and $\tilde{d}$-radius $\eta$. Choose $m \geq 1$ such that $1 /(m+1)<\eta<1 / m$. We claim that

$$
\Phi(x) \cap B \neq \varnothing \leftrightarrow\left(\forall s \in S_{m}\right)\left(h^{*}\left(2^{\omega}\right) \cap P(s) \neq \varnothing \rightarrow F(x) \cap P(s) \neq \varnothing\right) .
$$

To see this, let $h \in \Phi(x) \cap B$, so that $h\left(2^{\omega}\right) \subseteq F(x)$ and $\tilde{d}\left(h, h^{*}\right)<\eta$. Suppose that $h^{*}\left(2^{\omega}\right) \cap P(s) \neq \varnothing$ for some fixed $s \in S_{m}$. So there is $u \in 2^{\omega}$ such that $h^{*}(u) \in P(s)$. But $d\left(h(u), h^{*}(u)\right)<\eta<1 / m$, hence $(h(u))_{i}=\left(h^{*}(u)\right)_{i}, i=0,1, \ldots, m-1$. Consequently, $h(u) \in F(x) \cap P(s)$, so that $F(x) \cap P(s) \neq \varnothing$. Conversely, assume that the right side of (1) holds. Fix $s \in S_{m}$ such that $h^{*}\left(2^{\omega}\right) \cap P(s) \neq \varnothing$. Plainly, $h^{*}\left(2^{\omega}\right) \cap P(s)$ is a homeomorph of $2^{\omega}$. By hypothesis, $F(x) \cap P(s) \neq \varnothing$ and hence is a nonempty perfect subset of $\omega^{\omega}$, since $F(x)$ is a perfect subset of $\omega^{\omega}$. So $F(x) \cap P(s)$ contains a homeomorph of $2^{\omega}$. Let $\varphi_{s}$ be a homeomorphism of $h^{*}\left(2^{\omega}\right) \cap P(s)$ into $F(x) \cap P(s)$. We now piece together the homeomorphisms $\varphi_{s}$ for all $s \in S_{m}$ such that $h^{*}\left(2^{\omega}\right) \cap P(s) \neq \varnothing$. Thus, define $\varphi: h^{*}\left(2^{\omega}\right) \rightarrow 2^{\omega}$ be setting $\varphi=\varphi_{s}$ on $h^{*}\left(2^{\omega}\right) \cap P(s)$ whenever $s \in S_{m}$ is such that $h^{*}\left(2^{\omega}\right) \cap P(s) \neq \varnothing$. It is clear that $\varphi$ is a homeomorphism on $h^{*}\left(2^{\omega}\right)$ into $F(x)$. Let $h=\varphi \circ h^{*}$, so that $h$ is a homeomorphism of $2^{\omega}$ into $F(x)$, that is, $h \in \Phi(x)$. For any $u \in 2^{\omega}$, choose $s \in S_{m}$ such that $h^{*}(u) \in P(s)$. Then $h(u)=\varphi\left(h^{*}(u)\right)=\varphi_{s}\left(h^{*}(u)\right) \in P(s)$, so that $\left(h^{*}(u)\right)_{i}=(h(u))_{i}, i-0,1, \ldots, m-1$, and hence $d\left(h(u), h^{*}(u)\right) \leq 1 /(m+1)$. Consequently, since $u$ is arbitrary, it follows that $\tilde{d}\left(h, h^{*}\right) \leq 1 /(m+1)<\eta$, that is, $h \in B$ and so $\Phi(x) \cap B \neq \varnothing$, establishing the equivalence (1). It follows immediately from (1) that $\Phi$ is a Borel measurable multifunction as $F$ is.

Thus, $\Phi$ is a Borel measurable , $G_{\delta}$-valued multifunction from $X$ to $H\left(2^{\omega}\right)$ such that $\operatorname{Graph}(\Phi)$ is Borel in $X \times H\left(2^{\omega}\right)$. Hence, by a selection theorem in [4], there is a Borel measurable function $\psi: X \rightarrow H\left(2^{\omega}\right)$ such that $\psi(x) \in \Phi(x)$ for each $x \in X$. Define $g: X \times 2^{\omega} \rightarrow 2^{\omega}$ by $g(x, y)=\psi(x)(y)$. Clearly, for each $x \in X, g(x, \cdot)=\psi(x)$ and so a homeomorphism on $2^{\omega}$ into $F(x)$. For fixed $y \in 2^{\omega}, g(\cdot, y)=e_{y} \circ \psi$, which is Borel measurable on $X$. This completes the proof. 
We now turn to the proof of Theorem 2, which is similar to that of Theorem 1, only simpler.

Proof of TheOREM 2. Define a multifunction $\Phi$ from $X$ to $H\left(2^{\omega}\right)$ as follows:

$$
\Phi(x)=\left\{h \in H\left(2^{\omega}\right): h\left(2^{\omega}\right)=F(x)\right\} .
$$

By hypothesis, $\Phi(x)$ is nonempty and, as is easily checked, $\Phi(x)$ is closed in $H\left(2^{\omega}\right)$. If $B$ is as in the proof of Theorem 1, one verifies as in Theorem 1 the equivalence

$$
\Phi(x) \cap B \neq \varnothing \leftrightarrow\left(\forall s \in S_{m}\right)\left(h^{*}\left(2^{\omega}\right) \cap P(s) \neq \varnothing \leftrightarrow F(x) \cap P(s) \neq \varnothing\right) .
$$

Since $F$ is a Borel measurable multifunction, it follows from (2) that so is $\Phi$.

Thus, $\Phi$ is a Borel measurable, closed-valued multifunction from $X$ to $H\left(2^{\omega}\right)$. Hence, by a selection theorem in [2], there is a Borel measurable function $\psi: X \rightarrow$ $H\left(2^{\omega}\right)$ such that $\psi(x) \in \Phi(x)$ for each $x \in X$. The proof is concluded by setting $g(x, y)=\psi(x)(y)$ for $x \in X, y \in 2^{\omega}$.

4. Concluding remarks. We observe that more precise results can be obtained by this method by invoking the relevant selection theorem if the multifunction $F$ in Theorems 1 and 2 is assumed to be Borel measurable of class $\xi$ for fixed $\xi>0$.

In greater detail, suppose in Theorem 1 that the multifunction $F$ is of class $\xi>0$, that is, $\{x \in X: F(x) \cap V \neq \varnothing\}$ is a Borel set of additive class $\xi$ in $X$ for each open subset $V$ of $\omega^{\omega}$. An easy computation shows that then $\operatorname{Graph}(F)$ is Borel of multiplicative class $\xi+1$ in $X \times 2^{\omega}$. It follows that $\operatorname{Graph}(\Phi)$ is Borel of multiplicative class $\xi+1$ in $X \times H\left(2^{\omega}\right)$, where $\Phi$ is defined as in the proof of Theorem 1. Furthermore, it follows from (1) that $\Phi$ is a multifunction of class $\xi$. Consequently, by a result in [3], there is a selector $\psi$ of class $\xi+\xi$ for the multifunction $\Phi$. If we set $g(x, y)=\psi(x)(y), x \in X, y \in 2^{\omega}$, then $g(x, \cdot)$ is a homeomorphism of $2^{\omega}$ into $F(x)$ for each $x \in X$, and $g(\cdot, y)$ is a function of class $\xi+\xi$ for each $y \in 2^{\omega}$. If we assume in addition that $\operatorname{Graph}(F)$ is closed in $X \times \omega^{\omega}$, then the preceding argument will show that $g$ can be defined so that $g(\cdot, y)$ is a function of class $\xi$ for each $y \in 2^{\omega}$.

Similarly, suppose in Theorem 2 that the multifunction $F$ is of class $\xi>0$. Then (2) will imply that the multifunction $\Phi$ in the proof of Theorem 2 is of class $\xi+1$, so that a selector $\psi$ of class $\xi+1$ can be obtained for $\Phi$ by virtue of the Kuratowski-Ryll-Nardzewski selection theorem [2]. Setting $g(x, y)=\psi(x)(y)$ for $x \in X, y \in 2^{\omega}$, we see that $g(x, \cdot)$ is a homeomorphism of $2^{\omega}$ and $F(x)$ for each $x \in X$, and $g(\cdot, y)$ is a function of class $\xi+1$ for each $y \in 2^{\omega}$.

\section{REFERENCES}

1. D. Cenzer and R. D. Mauldin, Measurable parametrizations and selections, Trans. Amer. Math. Soc. 245 (1978), 399-408.

2. K. Kuratowski and C. Ryll-Nardzewski, A general theorem on selectors, Bull. Acad. Polon. Sci. Math. Astron. Phys. 13 (1965), 397403.

3. S. Levi and A. Maitra, Borel measurable images of Polish spaces, Proc. Amer. Math. Soc. 92 (1984), 98-102.

4. A. Maitra, Selectors for Borel sets with large sections, Proc. Amer. Math. Soc. 89 (1983), 705-708.

5. A. Maitra and V. V. Srivatsa, Parametrizations of Borel sets with large sections, Proc. Amer. Math. Soc. 93 (1985), $543-548$.

6. R. D. Mauldin, Borel parametrizations, Trans. Amer. Math. Soc. 250 (1979), 223234. 
7. R. Pol, A remark about measurable parametrizations, Proc. Amer. Math. Soc. 93 (1985), 628632.

8. R. Purves, Bimeasurable functions, Fund. Math. 58 (1966), 149-157.

Department of Mathematics and Computer Science, University of Miami, CORAL GABLES, Florida 33124

Indian Statistical Institute, 203 B.T. RoAd, Calcutta 700035, India (Current address of B. V. Rao and V. V. Srivatsa)

Current address (A. Maitra): Department of Theoretical Statistics, University of Minnesota, Minneapolis, Minnesota 55455 